\title{
Pilot Contamination Mitigation in Massive MIMO Systems-based 5G network
}

\author{
Nabeel Ali Abdullah \\ Public Authority for Applied Education and Training \\ Higher Institute for Telecommunication Navigation \\ Kuwait
}

\begin{abstract}
$5 G$, a future descent cellular network is predictable to encourage the increasing demand for continuous rising mobile users and the egression of services like the internet of things. Enormous MIMO has been specified as a promising technology that presents important improvements in the efficiency of spectral over $4 G$ technologies. Although, pilot contamination is the prime restricted factor while considering the TDD protocol. Several manners have been offered in literature to diminish the influence of pilot contamination in mobile systems. This paper provides an overview of the proposed a spatiotemporal approach which can mitigate the effect of pilot contamination by avoiding time-domain transmitting synchronous pilot and then a pilot allocation algorithm in the spatial domain. The proposed scenario provides a reduced level of intercell interference, which eventually gives it the ability to enhance the minimum and the mean achievable strength per terminal.
\end{abstract}

Key Words: Large-scale MIMO, $5 G$ Wireless Systems, Pilot contamination, Inter-cell interference.

\section{INTRODUCTION}

The fifth Cellular wireless networks are tracking to the future fifth-generation that demand a huge capacity system and greater data rates than the bygone generations. It has been shown in many researches that massive multiple input multiple output (MIMO) technology can meet the requirements of the 5G [1-3].

Massive MIMO is an Evolvability model of the multi-user MIMO technology, in which a base station (BS) equipped with many antennas serves many terminals at the same time and using the same time and same frequency, but in massive MIMO the antenna number's at the BS is much greater than the number of supplied terminals and each of the terminals connected to a single antenna. It is capable of enhancing the system spectral and energy efficiencies even using simple processing techniques [4,5]. Massive MIMO differentiates between different terminals through their propagation channels as they all use the same time and frequency resources in data communication, thus each BS must estimate the channels of its terminals. By using the time division duplex (TDD) mode and assuming channel reciprocity, the channel state information (CSI) for the uplink (UL) channel can be gotten through pilot sequence transmission from the terminal to the BS and utilized also for the downlink (DL) channel.

The CSI must be updated every coherence interval, which has only a limited number of orthogonal pilot sequences. We can deal with this situation by reusing the same pilot sequences set among the system cells, but simultaneous transmission of the same pilot sequence by different terminals causes BS interference as described in Figure 1, which is defined as pilot contamination [5-7]. The performance of large-scale MIMO is limited by the pilot contamination problem, which is argued in Section III, thus this problem must be diminished.

It has been shown that raising the number of antennas at the BS, increases the benefits of massive MIMO and overcomes many failures such as fast fading, uncorrelated noise, and intra-cell interference, but the inter-cell interference remains existing even if we used an unlimited number of antennas at the BS, which is the lead to pilot contamination [6].

In this paper, we suggest a scenario to diminish the level of inter-cell interference by decreasing the number of interfering cells at the pilot transmission phase and to enhance the performance of the low channel quality terminals by making use of the fact that large scale fading factors change slowly with time and can be considered unchanged over many coherence times, while assigning the pilot sequences to the terminals.

In the remainder of this article, firstly we review literature and categorize the schemes that try to mitigate the pilot contamination problem in Sec. II. Then, we show our system that is built to be a one of many cells and show how the problem of pilot 
contamination can affect its performance in the TDD mode in Sec. III. Then, our proposed scenario for pilot contamination mitigation is shown in Sec. IV. Then, the performance of our proposed scenario is evaluated in Sec. V.

\section{RELATED WORK}

The pilot contamination is a substantial challenge that hampers the deployment of massive MIMO technology in the $5 \mathrm{G}$ wireless communication systems with its full powers. Due to its importance, it has been heavily addressed in literature. We classify the schemes in literature from the pilot sequences allocation perspective into two categories:

\section{A. Schemes use random pilot allocation}

The papers [8,9] propose a time shifted protocol (TSP), which divides the cells to groups. Each group send their pilot sequences in non-overlapping times with respect to the other groups. It has been demonstrated that when we used a massive number of the BS antennas $(\rightarrow \infty)$, the cells of the same group only interfere with each other. This proposed protocol reduces the effect of pilot contamination, but it treats all the terminals equally and does not differentiate between them according to their channel qualities.

In [10] presents a pilot contamination precoding scheme which relies on the cooperation between the system BSs to diminish the influence of pilot contamination. It has been shown that this scheme is able to reduce the influence of pilot contamination completely when the BS antenna numbers is very large $(\rightarrow \infty)$, but practically this scheme is only useful when the number of antennas is small due to the information exchange overhead that increases with increasing the number of antennas.

\section{B. Schemes use pilot allocation algorithms}

In $[11,12]$, pilot sequences allocation algorithms are proposed to mitigate the effect of pilot contamination. These algorithms are based on exploiting the possibility to obtain and track the large-scale fading coefficient and using it as an indicator for the propagation quality.

In [11], for the purpose of assigning the pilot sequences to the terminals of a target cell, the terminals of this cell are sorted according to their channel quality, after that, the pilot sequences are divided according to the grade of interference created by receiving the pilot sequence from the other cells, finally the terminal with lower channel quality is assigned a pilot sequence with low interference level in a sequential order. It has been shown that this algorithm can maximize the minimum achievable rate, but it does not decrease the number of interfering cells.

In [12], another algorithm is proposed that outperforms the one in [11], but it also does not decrease the number of interfering cells and all the system cells interfere hath each other.

\section{SYSTEM MODELING \& PROBLEM DESCRIPTION}

Our system is modeled in this section influenced by the models of $[5,6]$ and using this model, we show how the inter-cell interference results from the pilot contamination.

\subsection{System Modeling}

We build the cellular system so that it has L cells, each one of radius R. The BS of each cell is occupied with an antenna array of many antennas elements $\mathrm{M}$ to supply many terminals $K \ll M$ that is equipped with a single antenna per terminal and evenly divided in the cell area as Figure 1 illustrated. All the BSs are attached to a central station and each BS supplies its terminals at the same time with the same bandwidth of $B(\mathrm{MHz})$.

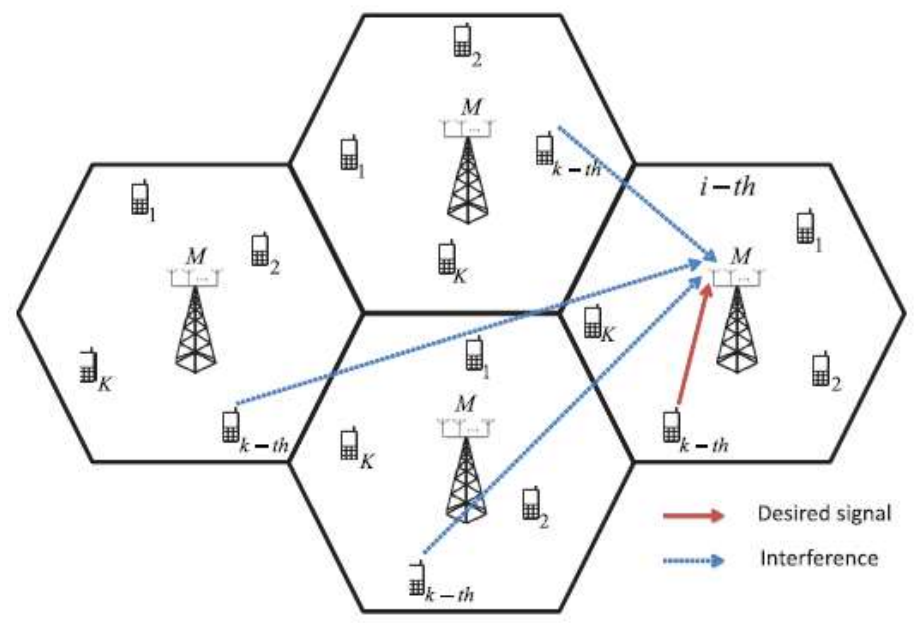

Figure 1. L cells of cellular system. 
The propagation channel between any terminal $k$ located in any cell $l$ and the $\mathrm{m}$-th antenna of the i-th BS can be modeled as given in [6] as follows:

$$
g_{m i k L}=h_{m i k L} \sqrt{\beta_{i k L}}
$$

where it is a consisted of small scale fading coefficient given by $h_{m i k L}$ and a large scale fading coefficient given by $\sqrt{\beta_{i k L}}$, which is the same for all the $\mathrm{M}$ antennas at the $\mathrm{i}$-th BS, because it a positive real number that embodies a range-dependent path loss and shadow fading that change slowly with distance. $h_{m i k L}$ changes every coherence time, but $\beta_{i k L}$ changes slowly with time and can be assumed constant for many coherence times. $\beta_{i k L}$ can be factorized as in [6] as follows:

$$
\beta_{i k L}=\frac{Z_{i k L}}{r_{i k L}^{y}}
$$

where $Z_{i k L}$ represents the shadow fading and it is known as a log-normal fading (the quantity $\left(10 \log _{10} Z_{i k L}\right)$ has a normal distribution), whose standard deviation can equal to $8 \mathrm{~dB}, r_{i k L}$ represents the distance between the terminal and the BS, while $\gamma$ is represent the path loss exponent, whose value can equal to 3.8 .

In our model, the used duplex mode is the TDD and we assume that the propagation channel is reciprocal, which means that we can estimate the channel in the UL and utilize it as an indication for the DL channel. We utilize Orthogonal Frequency-Division Multiplexing (OFDM) and the channel is presumed to be constant for T OFDM symbols, which symbolize the coherence time and $N$ subcarriers, which symbolize the coherence bandwidth.

The coherence time is scheduled as follows. Firstly, the terminals send UL pilot sequences for a duration of $\tau$ OFDM symbols out of the total of $T$, then the BS processes the received pilot sequences to estimate the channels, finally it uses the estimated channels to detect the UL data and precode the DL data. The matrix of orthogonal pilot sequences that are available for every coherence interval is the $\tau \times K$ matrix given by: $\left(\Phi=\left(\Psi_{1}, \Psi_{2}, \ldots, \Psi_{K}\right)\right)$, where each sequence has length $\tau$ and the number of sequences is $K=\tau N$.

\subsection{Problem Description}

Now, we have a system of $L$ cells, each of $K$ terminals (which equals the largest number of available pilots) and if we assume simultaneous pilot transmission, this means that we need $L \times K$ orthogonal pilot sequences for the purpose of good channel estimation, but the available is only $K$, thus either we decrease the total number of terminals served by the system to only $K$, which results in a very low system capacity (this is rejected solution, because it does not meet the $5 \mathrm{G}$ requirements) or we have to reuse the same orthogonal matrix $\phi$ in all the system cells, which results in pilot contamination.

\subsubsection{Pilot Contamination}

Having each terminal transmitted its assigned pilot, the BS of the $i$-th cell receives the orthogonal matrix of pilot sequences not only from its own terminals, but also from the terminals of all the other cells as shown in Figure 1 for the K-th pilot sequence only for clarity. The $M \times K$ matrix represent The channel coefficients between the terminals of any cell $l$ and the $i$-th cell:

$$
G_{i l}=H_{i l} D_{i l}^{0.5}
$$

where $H_{i l}$ is $M \times K$ matrix of small-scale fading, whose elements have the same standard normal distribution and are mutually independent, $D_{i l}$ and is $K \times K$ diagonal matrix of large-scale fading $\beta_{i k L}, k=1, \ldots, K$.

The BS of the $i$-th cell receives the following $M \times \tau$ matrix [5]:

$$
Y_{i}=\sqrt{P_{P}} \sum_{l=1}^{L} G_{i l} \phi^{T}+W_{i}
$$

where $P_{P}$ is the power used by each terminal in pilot transmission, $G_{i l}$ is the matrix of channel coefficients between the 1-th cell terminals and the i-th BS, T means a matrix transpose and $W_{i}$ is the $M \times \tau$ matrix that represents additive white noise at receiver. The elements of $W_{i}$ have the same standard normal distribution and are mutually independent, and they are also independent with the elements of $G_{i l}$.

The matrix of channel coefficients of the $i$-th cell can be estimated by its BS as in [5] as follows: 


$$
\overline{G_{l l}}=\frac{1}{\sqrt{P_{P}} \tau} Y_{i l} \phi^{*}=G_{i i}+\sum_{l \neq i}^{L} G_{i l}+\frac{1}{\sqrt{P_{P}} \tau} W_{i} \phi^{*}
$$

Where $*$ means a matrix conjugate and $\tau$ is the length of each pilot sequence. By looking at this equation and comparing it with Equation 3, we note that the estimated matrix is not the actual channel matrix of the $i$-th BS, but it is a sum of all the channel matrices from all the system cells that use the same orthogonal matrix of pilot sequences to the $i$-th BS, in addition to noise. Transmitting the same pilot sequence by more than one terminal, results in the contamination of the estimated channel of each terminal of them by the channels of another terminals. Using this contaminated estimated channel matrix in detecting the data symbols that will be transmitted from the $i$-th cell terminals, will result in inter-cell interference. This matter is debated in the next subsection.

\subsubsection{Inter-cell Interference}

Having the channel estimation process been performed by each BS, each terminal can transmit its data symbol. Having each terminal transmitted its data symbol, the BS of the $i$-th cell receives the following $M \times l$ vector of symbols:

$$
y_{i}=\sqrt{P_{d}} \sum_{l=1}^{L} G_{i l} x_{l}+\omega_{i}
$$

where $P_{d}$ is the power per terminal used in data transmission, $x_{l}$ is the $K \times l$ vector of Gaussian data symbols from terminals of the $l$-th cell, and $\omega_{i}$ is the $M \times l$ vector that represents the added noise at the receiver. The elements of $\omega_{i}$ have the same standard normal distribution and are mutually independent.

Using maximum ratio combining as the signal detection technique, the estimated data vector by the $i$-th BS is given as follows:

$$
\widehat{x}_{\imath}=\frac{1}{\sqrt{P_{d}} M} \widehat{G_{l l}^{H}} y_{i}
$$

where ${ }^{H}$ is used to denote the matrix conjugate transpose (Hermitian) as in [14] .

According to [6] and following a mathematical derivation as in [13], when the number of antennas tends to infinity, Equation 7 becomes:

$$
\widehat{x}_{l}=\sum_{l=1}^{L} D_{i l} x_{l}
$$

By looking at the previous equation, we find that the term that represents the fast fading $H_{i l}$ and also the term that represents the noise disappear, and if we take the $k$-th component of $\widehat{x}_{l}$, it can be written as:

$$
\widehat{x_{k l}}=\sum_{l=1}^{L} \beta_{i k l} x_{k l}=\beta_{i k i} x_{k i}+\sum_{l \neq i}^{L} \beta_{i k l} x_{k l}
$$

This estimated data symbol is not just the transmitted symbol by the $k$-th terminal of the $i$-th cell. Instead, it is the sum of all the transmitted symbols from the terminals that have been assigned the same pilot sequence. Thus, we can conclude that pilot contamination results in wrong channel estimation in each cell, that considers the combination of channels from all the $k$-th terminals of different cells to its BS as the channel of its own $k$-th terminal, which in turn causes that the BS not only receives the data symbol of its own $k$-th terminal, which is the desired signal and reject any other symbol, but also receives data symbols from the $k$-th terminals of other cells, which represents the interference. so, we can represent the signal to interference and noise ratio (SINR) of any terminal $k$ in the $i$-th cell as:

$$
\operatorname{SINR}_{k}=\frac{\left(\beta_{i k i}\right)^{2}}{\sum_{l \neq i}^{L}\left(\beta_{i k i}\right)^{2}}
$$

\section{PROPOSED SCENARIO}

Our proposed scenario targets mitigating the pilot contamination effect and enhancing the performance of the low channel quality terminals by avoiding synchronous pilot transmission and following a pilot allocation algorithm instead of assigning the pilot sequences randomly to the terminals. 


\subsection{Asynchronous Pilot Transmission (APT)}

Under the assumption of synchronous pilot sequences transmission (SPT), adopting a reuse factor of 3 instead of 1 improves the SINR of all terminals as it decreases the number of interfering cells to one third [6] as shown in Figure 2(a), but this comes at the cost of decreasing the available bandwidth in each cell to one third, which in turn results in a lower per terminal capacity of nearly half of the terminals [6] as shown in Figure 2(b), which finally results in a lower system capacity compared to the case of reuse factor of 1. Adopting APT, which can be achieved through using the TSP proposed in [9] enables us to take the SINR improvement achieved by the reuse factor of 3 in the SPT scenario without decreasing the available bandwidth in each cell, which results in a better per terminal capacity than a reuse factor of 1 in the SPT scenario as shown in Figure 2(b).

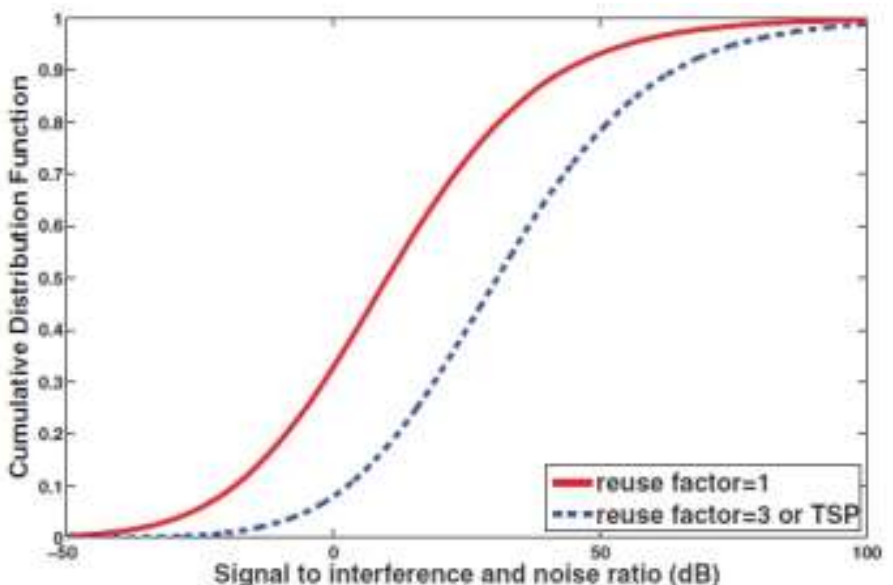

(a)

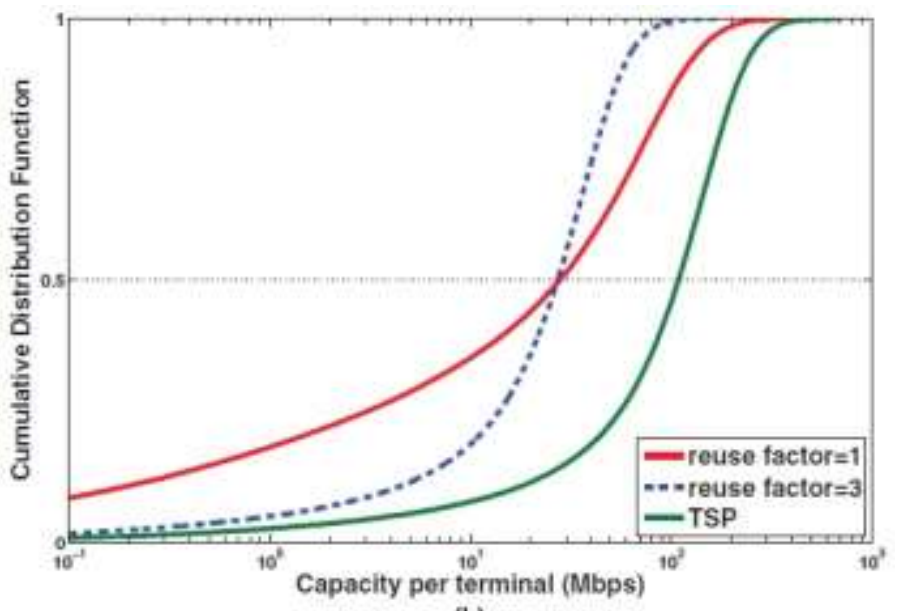

(b)

Figure 2. (a) The cumulative distribution function of the SINR for different schemes. (b) The cumulative distribution function of the capacity per terminal for different schemes.

\subsection{Pilot Allocation Algorithm (PAA)}

Instead of assigning the pilot sequences to the terminals randomly regardless of their channel quality, we can use a PAA. PAAs proposed in [11,12], show improved performance compared to the random pilot allocation. these PAAs are based on the availability of large-scale fading factors, which are easily to be obtained and tracked according to [10-13]. The PAA of [12] shows also an improvement compared to that of [11]. Using the PAA of [12], the large-scale fading factors from all terminals to each BS are made available at a network hub. To improve the minimal achieved rate by a target cell terminals, the network hub assigns the pilot sequences to the system terminals so that, the same pilot sequence assigned to the terminal of low channel quality in the target cell, is assigned to the terminals of low inter-cell interference on the target cell in the other cells.

\subsection{Proposed Scheme}

We can benefit from the previously discussed schemes, if we integrate them into one scheme through a spatiotemporal scenario as follows:

- We partition The system into 3 groups: $G_{1}, G_{2}$, and $G_{3}$ through using 3 different time protocols as displayed in Figure 3 .

- Each cell determines the large-scale fading factors from all terminals to its BS and sends them to the central station.

- In the central station, for a target cell $(i)$ :

- It uses the parameter $\left(\alpha_{k}=\left(\beta_{i k i}\right)^{2}, k=1,2, \ldots, K\right)$ as an indicator to the channel quality of the terminal $k$ of this cell.

- It uses the parameter $\left(\mu_{k l}=\left(\beta_{i k l}\right)^{2}, k=1,2, \ldots, K\right.$ and $\left.l=1,2, \ldots . L, l \neq i\right)$ as an indicator to the inter-cell interference level of the $k$-th terminal of the $l$-th cell.

○ The terminals of the $i$-th cell are sorted according to their channel quality, so that: $\alpha_{1}<\alpha_{2}<\ldots<\alpha_{k}$.

$\circ \quad$ the terminals of the other cells are sorted according to their interference on the target, so that: $\mu_{1 l}<$ $\mu_{2 l}<\ldots .<\mu_{k l}$.

- The pilot sequences are assigned to the system terminals so that, the same pilot sequence assigned to a terminal in the target cell, is assigned to the corresponding terminals of all the other cells in a sequential order: $\psi_{1}, \psi_{2}, \ldots, \psi_{k}$.

Now, a low channel quality terminal located in a target cell only interferes with the terminals that cause low interference level to the target cell located in the other cells of the target cell group, and a high channel quality terminal located in a target cell only interferes with the terminals that cause high interference level to the target cell located in the other cells of the target cell group. This is rather than all the cells as is the case in [12]. Thus, our scenario modifies the SINR per terminal to that of the following equation: 
International Journal of Advances in Scientific Research and Engineering (ijasre), Vol 6 (3), March -2020

$$
\operatorname{SINR}_{k}=\frac{\alpha_{k}}{\sum_{l \in G_{1}, l \neq i} \mu_{k l}}
$$

By knowing the SINR per terminal, we can find the capacity per terminal in megabits per second (Mbps) using Shannon relation according as follows:

$$
C_{k}=(B)\left(\left(\frac{T-\tau-n}{T}\right)\left(\left(\frac{T_{s}-T_{c}}{T_{c}}\right) \log _{2}\left(1+\operatorname{SINR}_{k}\right)\right)\right)
$$

where $n$ is the number of OFDM symbols wasted in signal processing operations at the BS, Ts is the duration of the symbol, and $T_{c}$ is the duration of the cyclic prefix. Ts equals $71.4 \mu$ seconds and $T_{c}$ equals $4.76 \mu$ seconds.

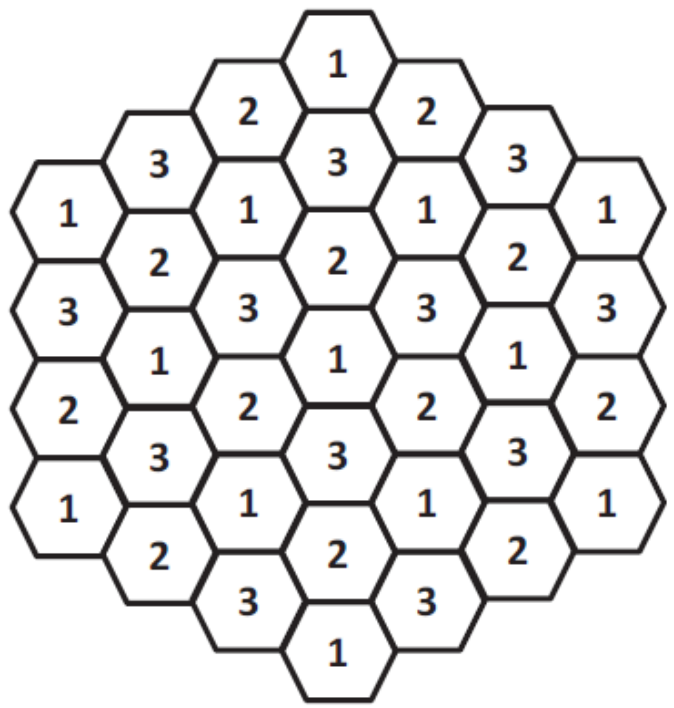

(a) Cellular system of 3 groups

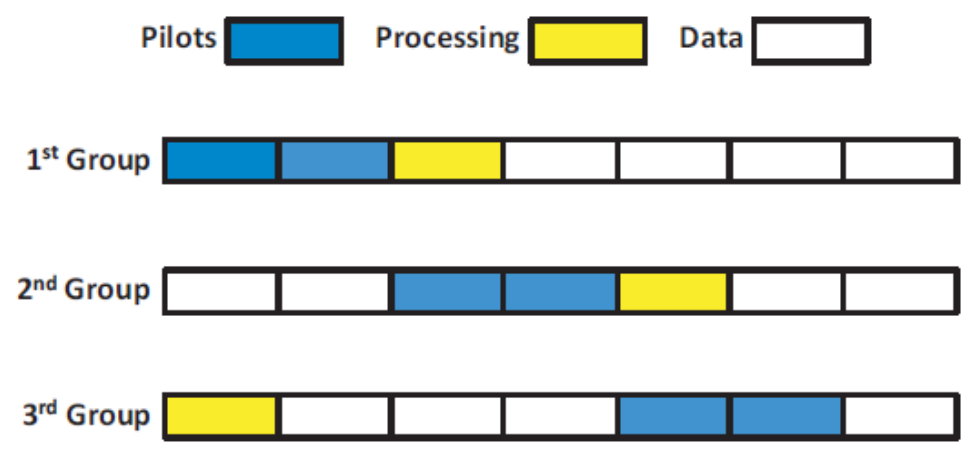

(b) Time shifted protocol

Figure 4. Division of our cellular system into 3 groups using a time shifted protocol.

\section{PERFORMANCE EVALUATION}

In this section, we evaluate the performance of our proposed scheme compared to other schemes: the conventional scheme (in which, we consider random pilot allocation and simultaneous transmission of identical pilots), the TSP scheme of [9], and the PAA scheme of [12] in terms of the minimum achievable capacity per terminal, the maximum achievable capacity per terminal, and the mean achievable capacity per terminal.

In evaluating our proposed scheme, we use computer simulations based on the previous equations presented throughout this article, and we assign values to the equations terms as in Table 1.

Table (1) Simulation Parameters.

\begin{tabular}{ll}
\hline parameter & value \\
\hline $\mathbf{T}_{\mathbf{s}}$ & $71.4 \mu \mathrm{s}$ \\
$\mathbf{T}_{\mathbf{c}}$ & $4.76 \mu \mathrm{s}$ \\
$\mathrm{L}$ & 37 cells \\
$\boldsymbol{R}$ & $160 \mathrm{~m}$ \\
$\mathbf{n}$ & 1 OFDM symbol \\
$\boldsymbol{\tau}$ & 2 OFDM symbols \\
$\mathrm{T}$ & 7 OFDM symbols \\
$\mathrm{B}$ & $20 \mathrm{MHz}$ \\
$\boldsymbol{N}$ & 14 subcarrier \\
\hline
\end{tabular}

In Figure 5, the cumulative distribution function (CDF) of the capacity per terminal for our proposed scheme is compared with those of the other schemes. Our proposed scheme provides a higher capacity per terminal for the whole time compared to the conventional and the PAA schemes. Our proposed scheme provides a higher capacity per terminal up to the 0.8 value of the CDF compared to the TSP scheme, then it provides a lower capacity per terminal. 


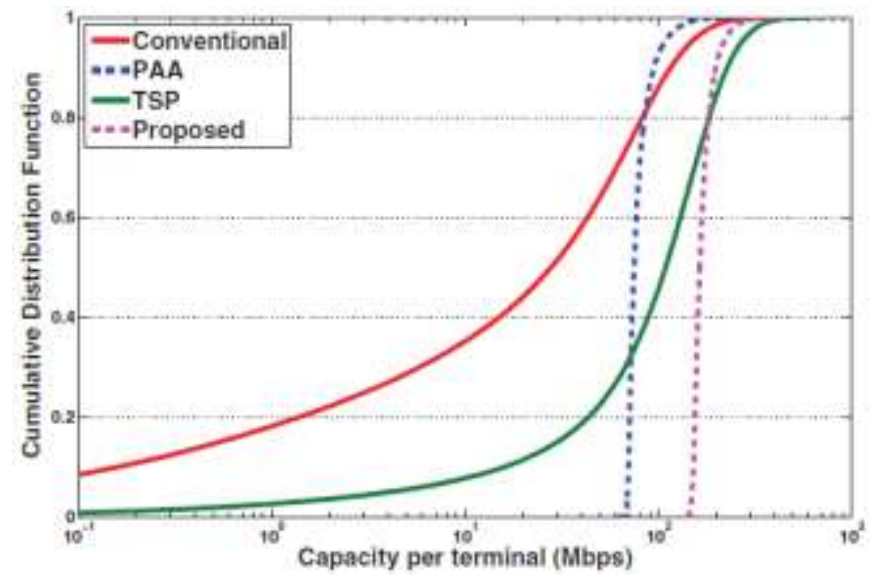

Figure 5. Cumulative distribution functions of capacity per terminal for our proposal and other schemes.

From Figure 5, we note also that: in terms of the minimum achievable capacity per terminal, our proposed scheme is the best one, because it decreases the number of interfering terminals compared to the conventional and the PAA schemes, and guarantees that the low channel quality terminal in the target cell interferes only with the other cells' terminals that cause low interference to the target cell compared to the conventional and the TSP schemes. In terms of the maximum achievable capacity per terminal, our proposed scheme outperforms the conventional and the PAA schemes, but the TSP scheme outperforms our proposed scheme, despite having the same number of interfering cells. This results from the fact that random pilot allocation in the TSP may cause that a terminal of high channel quality interferes with terminals causing low interference.

\section{Conclusions}

This research paper debate the case of pilot contamination that is treated as the performance limitation of the massive MIMO systems. The researchers presented our proposed scenario to combat the effect of this issue. Our scenario reduces the interference by a factor equals to the number of the system groups. Additionally, it enhances the terminals of low channel quality. We have proved through the simulations presented, the ability of our scenario to improve the minimum achievable capacity per terminal and enhance the system spectral efficiency through increasing the mean capacity per terminal without increasing the system bandwidth.

\section{REFERENCES}

1. C.-X. Wang et al., "Cellular architecture and key technologies for 5G wireless communication networks," IEEE Communications Magazine, vol. 52, no. 2, pp. 122-130, Feb. 2014.

2. J. G. Andrews et al., "What will 5G be?," IEEE Journal on Selected Areas in Communications, vol. 32, no. 6, pp. 1065 1082, Jun. 2014.

3. F. Boccardi, R. W. Heath, A. Lozano, T. L. Marzetta, and P. Popovski, "Five disruptive technology directions for 5G," IEEE Communications Magazine, vol. 52, no. 2, pp. 74-80, Feb. 2014.

4. E. G. Larsson, O. Edfors, F. Tufvesson, and T. L. Marzetta, "Massive MIMO for next generation wireless systems," IEEE Communications Magazine, Vol. 52, no. 2, pp. 186-195, Feb. 2014.

5. L. Lu, G. Y. Li, A. L. Swindlehurst, A. Ashikhmin, and R. Zhang, "An overview of massive MIMO: Benefits and challenges," IEEE Journal of Selected Topics in Signal Processing, vol. 8, no. 5, pp. 742-758, Oct. 2014.

6. T. L. Marzetta, "Noncooperative cellular wireless with unlimited numbers of base station antennas," IEEE Transactions on Wireless Communications, vol. 9, no. 11, pp. 3590-3600, Nov. 2010.

7. K. Zheng et al., "Survey of large-scale MIMO systems," IEEE Communications Surveys and Tutorials, vol. 17, no. 3, pp. 1738-1760, Apr. 2015.

8. F. Fernandes, A. Ashikhmin, and T. L. Marzetta, "Interference reduction on cellular networks with large antenna arrays," in Proc. IEEE International Conference on Communications (ICC), Ottawa, Jun. 2012.

9. F. Fernandes, A. Ashikhmin, and T. L. Marzetta, "Inter-cell interference in noncooperative TDD large scale antenna systems," IEEE Journal on Selected Areas in Communications, vol. 31, no. 2, pp. 192-201, Feb. 2013.

10. A. Ashikhmin and T. Marzetta, "Pilot contamination precoding in multicell large scale antenna systems," in Proc. IEEE International Symposium on Information Theory (ISIT), Cambridge, Jul. 2012.

11. X. Zhu, Z. Wang, L. Dai, and C. Qian, "Smart pilot assignment for massive MIMO," IEEE Communications Letters, vol. 19, no. 9, pp. 16441647, Sept. 2015.

12. M. Alkhaled, E. Alsusa, and K. A. Hamdi, "A new allocation algorithm for pilot contamination mitigation in TDD massive MIMO systems," in Proc. IEEE Wireless Communications and Networking Conference (WCNC), San Francisco, Mar. 2017. 
International Journal of Advances in Scientific Research and Engineering (ijasre), Vol 6 (3), March -2020

13. M. Alkhaled, E. Alsusa, and K. A. Hamdi, "Adaptive pilot allocation algorithm for pilot contamination mitigation in TDD massive MIMO systems," in Proc. IEEE Wireless Communications and Networking Conference (WCNC), San Francisco, Mar. 2017.

\section{AUTHOR'S PROFILE}

Nabeel A. M Abdullah

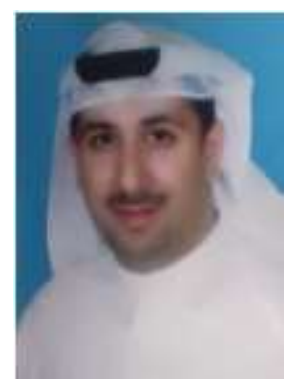

Received his B.Sc. in electrical engineering from the University of Kuwait in 1996, then he worked in many Telecommunication posts. He worked as Training Programs Developer, Training and Academic Program Facilitator at Public Authority for Applied Education \& Training PAAET, Department Head at the Curriculum Development Center (CDC) 2010-2011, and Transmission Department Head in Higher Institute of Telecommunication \& Navigation (HITN) (PAAET) 2011-2016. Currently specialized instructor in HITN PAAET. 